\title{
Binary Evolution Models with Rotation
}

\author{
N. Langer, S.-C. Yoon, J. Petrovic \\ Astronomical Institute, P.O. Box 80000, NL-3508 TA Utrecht, The \\ Netherlands
}
A. Heger
Department of Astronomy and Astrophysics, Enrico Fermi Institute, The University of Chicago, 5640 S. Ellis Avenue, Chicago IL 60637, $U S A$

\begin{abstract}
We discuss the first available binary evolution models which include up-to-date rotational physics for both components, as well as angular momentum accretion and spin-orbit coupling. These models allow a self-consistent computation of the mass transfer efficiency during Roche-lobe overflow phases, and a determination of the transition from quasi-conservative to non-conservative evolution. Applications to massive binary systems lead to predictions for the spin rates of compact objects in binaries, and for the occurrence of gamma-ray bursts from collapsars in binaries. Rotational effects in accreting white dwarfs are found to stabilise the shell burning and decrease the carbon abundance in progenitor models for Chandrasekhar-mass Type Ia supernovae, and to potentially avoid a detonation of the white dwarf within the sub-Chandrasekhar mass scenario.
\end{abstract}

\section{Introduction}

The evolution of a single star can be strongly influenced by its rotation (e.g. Heger \& Langer 2000; Meynet \& Maeder 2000), and evolutionary models of rotating stars are now available for many masses and metallicities. While the treatment of the rotational processes in these models is not yet in a final stage (e.g. magnetic dynamo processes are just about to be included; Heger et al., this volume), they provide first ideas of what rotation can really do to a star.

Effects of rotation, as important they are in single stars, can be much stronger in the components of close binary systems: Estimates of the angular momentum gain of the accreting star in mass transferring binaries show that critical rotation may be reached quickly (Packet 1981; Langer et al. 2000). Therefore, we need binary evolution models which include a detailed treatment of rotation in the stellar interior, as in recent single star models. However, in binaries, tidal processes as well as angular momentum accretion need to be considered at the same time. Some first such models are now available and are discussed below.

These models provide evidence for rotational processes in binaries being essential for some of the most exciting cosmic phenomena, which may occur exclusively in binaries: Type Ia supernovae, the main producers of iron and 
cosmic yardstick to measure the accelerated expansion of the universe. Gammaray bursts from collapsars - which current stellar models with rotation preclude to occur in single stars (Heger et al., this volume; Woosley \& Heger, this volume) - may provide the most powerful explosions in the universe and trace star formation to its edge. And black holes, even though also formed by single stars, need a companion to become "visible".

\section{How much matter can stars accrete from a binary companion?}

As mentioned above, non-magnetic accretion, i.e. accretion via a viscous disk or via ballistic impact, transports angular momentum and can lead to a strong spin-up of the mass gaining star. For disk accretion, it appears plausible that the specific angular momentum of the accreted matter corresponds to Keplerrotation at the stellar equator; this leads to a spin-up of the whole star to critical rotation when its initial mass is increased by about $20 \%$ (e.g. Packet 1981). Can accretion continue beyond this?

Theoretically, this appears possible, as viscous processes may transport angular momentum outwards through the star, the boundary layer, and the accretion disc (e.g. Paczynski 1991). However, as the star may be strongly rotating, its wind mass loss may be dramatically increased (Langer 1997, 1998), which may render the mass transfer process inefficient.

Observations of massive post-mass transfer binary systems constrain this effect. Table 1 lists parameters of four different kinds of massive close binary systems which give opposite answers. The two $\mathrm{O}$ stars in the Case A binary (mass transfer starts while both stars undergo core hydrogen burning) V 729 Cyg have a mass ratio of 3.5 but the same spectral type and visual flux. Clearly, an initial mass ratio close to 1 is required to get close to the observed current mass ratio. However, as during Case A the primary star (as we designate the initially more massive star in a binary) loses just about half of its mass, a mass ratio of at most 2 could be produced were the secondary (the initially less massive star in a binary) not allowed to accrete. Another system showing strong evidence for accretion is the massive X-ray binary Wray 977; it would require that stars of $\gtrsim 40 \mathrm{M}_{\odot}$ form neutron stars to explain this system without accretion (Wellstein \& Langer 1999).

Several Galactic short period WNE+O binaries, on the other hand, can not be understood had the $\mathrm{O}$ star accreted substantial amounts from the WNE progenitor (Petrovic \& Langer 2002). While those might have formed through common envelope evolution - for which little accretion is expected - the key $\mathrm{X}$-ray binary $4 \mathrm{U} 1700-37$ has such a short period that a major accretion phase can be excluded. However, as Case $\mathrm{C}$ evolution (mass transfer starts after core helium burning of the primary star) would lead to a compact object much more massive than $2.4 \mathrm{M}_{\odot}$, Case $\mathrm{B}$ evolution (mass transfer starts just after core hydrogen burning of the primary star) is most likely here (Clark et al. 2002).

We conclude from these observed binary systems that in some massive close (Case A or B) binaries, the mass transfer process is nearly conservative, while in others it is strongly non-conservative. In the following, we show that including rotational physics into binary evolution calculations allows to recover both features within the same physical model. 
Table 1. Clues on accretions efficiencies from observed binaries

\begin{tabular}{ccccc}
\hline object & sp. types & orb. period & masses/ratio & accretion? \\
\hline V729 Cyg $^{1}$ & O7+O7 & $6.6 \mathrm{~d}$ & $q=3.5$ & YES \\
${\text { Wray } 977^{3}}^{2}$ & BI+NS & $44 \mathrm{~d}$ & $40 \mathrm{M}_{\odot}+1.4 \mathrm{M}_{\odot}$ & YES \\
3 systems & WNE+O & $\sim 8 \mathrm{~d}$ & $q \simeq 0.5$ & $\mathrm{NO}^{5}$ \\
$4 \mathrm{U} 1700-37^{6}$ & O6I+NS/BH & $3.4 \mathrm{~d}$ & $58 \mathrm{M}_{\odot}+2.4 \mathrm{M}_{\odot}$ & $\mathrm{NO}^{5}$ \\
\hline \hline
\end{tabular}

${ }^{1}$ Bohannan \& Conti (1976)

${ }^{2}$ both components have the same visual magnitude

${ }^{3}$ Kaper et al. (1995), Wellstein \& Langer (1999)

${ }^{4}$ Petrovic \& Langer (2002)

5 meaning: $10 \%$ or less

${ }^{6}$ Clark et al. (2002)

\section{Binary models with rotating components}

We have constructed binary evolution models using the code of Wellstein et al. (2001), but including the physics of rotation as in the single star models of Heger et al. (2000) for both components. In addition, spin-orbit coupling according to Zahn (1977) has been added, and rotationally enhanced winds are implemented as in Langer (1998). The specific angular momentum of the accreted matter is assumed to be that of Kepler rotation at the stellar equator in the case of disk accretion, and determined by integrating the equation of motion of a test particle in the Roche potential in case the accretion stream impacts directly on the secondary star (Wellstein 2001).

Fig. 1 shows the evolution of the equatorial rotation velocity in a system starting out with a $16 \mathrm{M}_{\odot}$ and a $15 \mathrm{M}_{\odot}$ star in a 3 day orbit. The initial rotational velocity of both stars is unimportant since they evolve quickly into rotation which is synchronous with the orbital revolution, due to spin-orbit coupling. Each of the three thermal time scale mass transfer phases through which this system evolves (rapid Case A, Case AB, and Case ABB; see Wellstein et al. 2001) leads to a strong spin-up of the secondary star and an equally drastic spin-down of the primary (see Langer 1998, for the purely mechanical spin-down effect).

The accretion induced spin-up can bring the secondary close to critical rotation and thus strong rotationally enhanced mass loss sets in. The difference between the mass overflow rate $-\dot{M}_{1}$ and the wind mass loss rate of the secondary is the net accretion rate $\dot{M}_{2}$ of the secondary star. Fig. 2 shows that during much of the rapid Case A mass transfer - during which the accreting star increases its mass from $15 \mathrm{M}_{\odot}$ to $\sim 23 \mathrm{M}_{\odot}-$, it is $-M_{1}=\dot{M}_{2}$. This is possible due to two factors: 1) During direct impact accretion, the specific angular momentum of the accreted material is only a fraction of the respective Kepler angular momentum. 2) As the secondary star fills a significant fraction of its Roche volume, tidal coupling removes part of the accreted angular momentum and feeds it into the orbit.

As the orbit widens significantly during the later evolution, the specific angular momentum of the accreted matter increases and tidal forces weaken. Thus, while the accretion efficiency is close to 1 during Case A, it is less than 0.1 


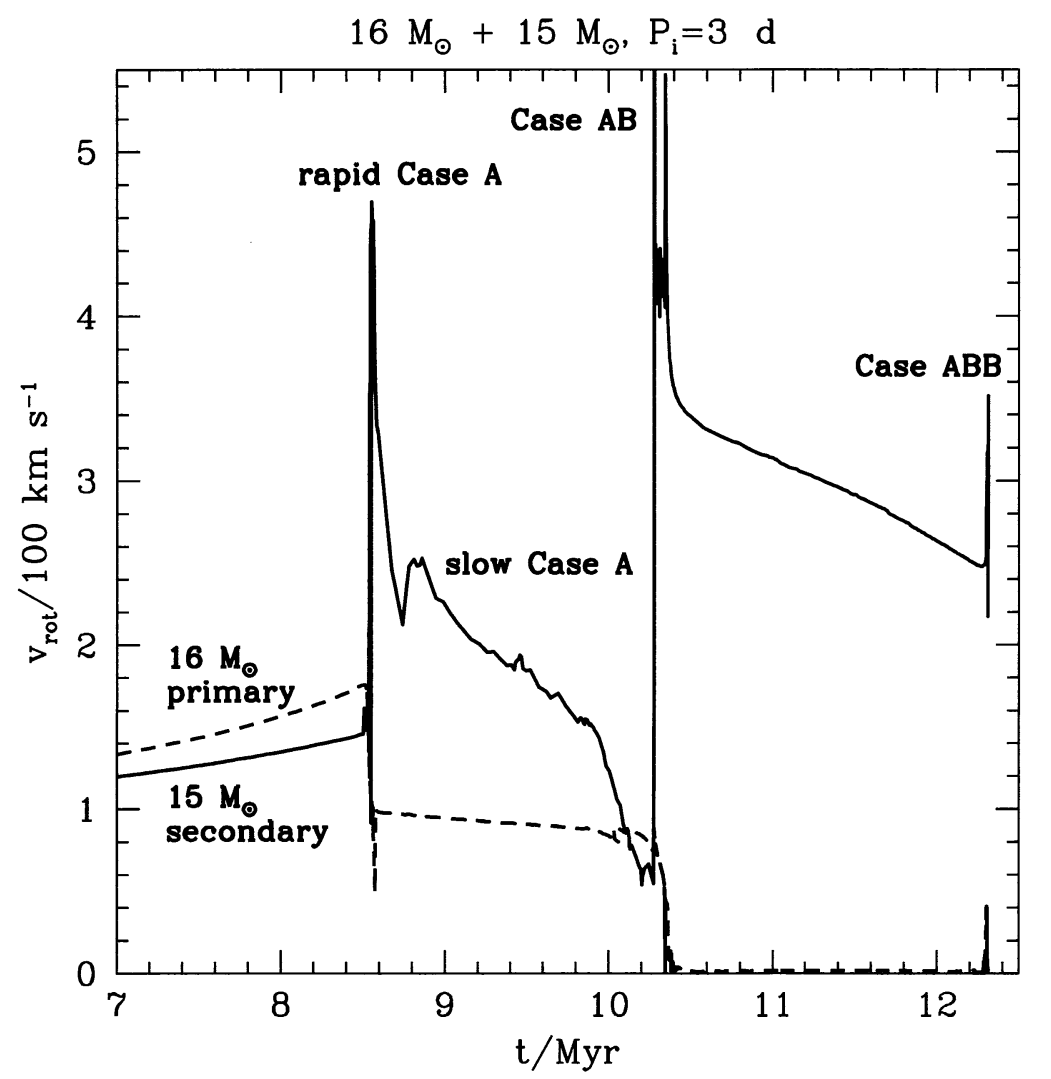

Figure 1. Equatorial rotation velocity for primary (dashed line) and secondary (solid line) component of a $16 \mathrm{M}_{\odot}+15 \mathrm{M}_{\odot}$ system with an initial orbital period of $3 \mathrm{~d}$ as function of time, starting at an age of $7 \mathrm{Myr}$, i.e. well before the onset of mass transfer, until the end of Case ABB mass transfer, which corresponds to the time of the supernova explosion of the primary. The four different mass transfer phases which occur in this system are indicated; except for the slow Case A mass transfer they occur on the thermal time scale of the primary star (see also Fig. 2).

later, resulting in a time average over the whole evolution of 0.67 . For systems which start out with a wider orbit, we find a low accretion efficiency throughout (Fig. 3). For $16 \mathrm{M}_{\odot}+15 \mathrm{M}_{\odot}$ systems, we find a critical initial period of $\sim 8 \mathrm{~d}$ beyond which the secondary accretes only little.

These models imply that, despite the angular momentum problem for the accretion star (Packet 1981), quasi-conservative evolution of massive close binaries is possible. However, already in early Case B systems, the accretion efficiency may be strongly reduced compared to binary models without rotation. 


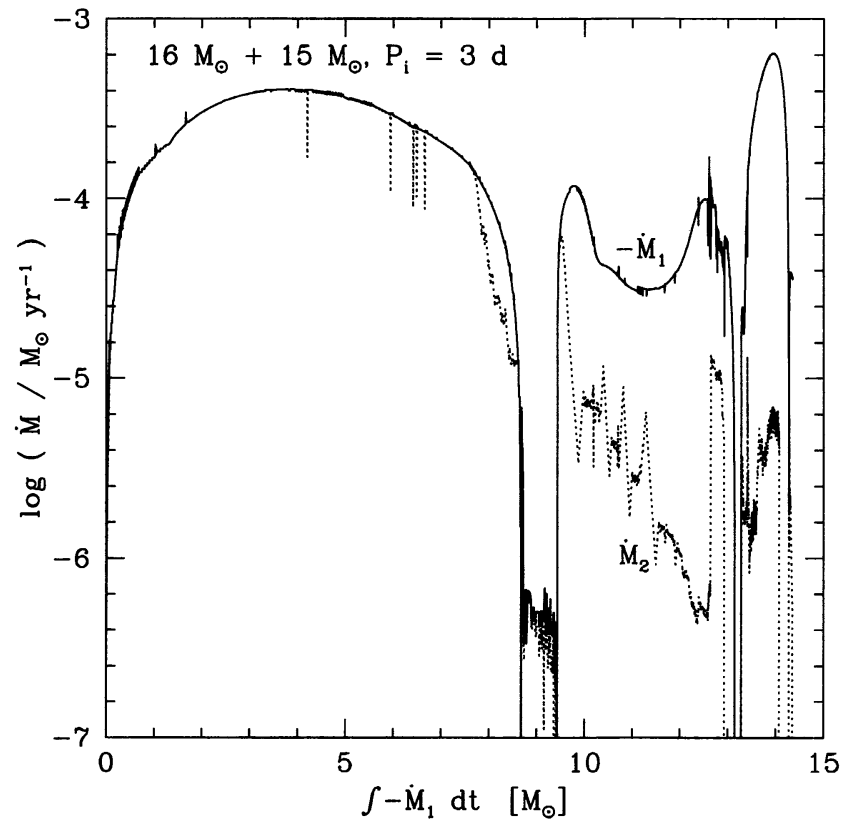

Figure 2. For the $16 \mathrm{M}_{\odot}+15 \mathrm{M}_{\odot}$ system with an initial orbital period of 3 days: mass transfer rate $\left(-\dot{M}_{1}\right.$; full drawn line) and mass accretion rate of the secondary $\left(\dot{M}_{2} ;\right.$ dotted line) as function of the total amount of mass which has already been transferred. Four discrete mass transfer phases can be distinguished: rapid Case $\mathrm{A}\left(-\dot{M}_{1} \gtrsim 10^{-4} \mathrm{M}_{\odot} \mathrm{yr}^{-1}, \Delta M_{1} \simeq 8.7 \mathrm{M}_{\odot}\right)$, slow Case A $\left(-\dot{M}_{1} \gtrsim 10^{-7} \mathrm{M}_{\odot} \mathrm{yr}^{-1}, \Delta M_{1} \simeq 0.8 \mathrm{M}_{\odot}\right)$, Case $\mathrm{AB}\left(-\dot{M}_{1} \gtrsim\right.$ $\left.10^{-5} \mathrm{M}_{\odot} \mathrm{yr}^{-1}, \Delta M_{1} \simeq 3.8 \mathrm{M}_{\odot}\right)$, and Case ABB $\left(-\dot{M}_{1} \gtrsim 10^{-4} \mathrm{M}_{\odot} \mathrm{yr}^{-1}\right.$, $\left.\Delta M_{1} \simeq 1.2 \mathrm{M}_{\odot}\right)$.

\section{Black hole formation in binaries}

While single stars more massive than $\sim 25 \mathrm{M}_{\odot}$ are supposed to form black holes (Fryer 2002), it is a challenge to binary evolution modellers to find a way to make black hole binaries - binary systems consisting of a non-degenerate star and a black hole. The reason is that the initially more massive star, which is supposed to end up as black hole, undergoes tremendous mass loss (Fig. 2, and see Wellstein \& Langer 1999).

Brown, Lee \& Bethe (1999) suggested the Case C channel to form the Galactic low mass BH binaries - a low mass star in close orbit with a black hole of typically $6 \mathrm{M}_{\odot}$ (Orosz 2002), i.e. starting with a wide system consisting of a (say) $25 \mathrm{M}_{\odot}$ and a $5 \mathrm{M}_{\odot}$ star. This suggestion is strongly supported by the massive post-Case B X-ray binary $4 \mathrm{U} 1700-37$ (Table 1): it shows that in Case B systems, even a $60 \mathrm{M}_{\odot}$ primary forms a compact object of only $2.4 \mathrm{M}_{\odot}($ Clark et al. 2002). 


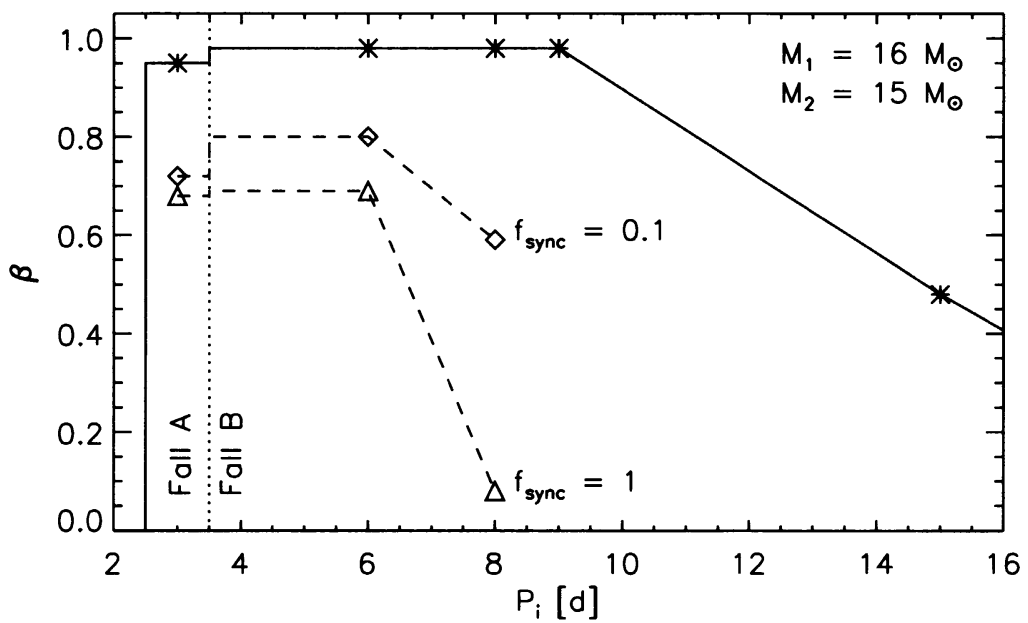

Figure 3. Time averaged mass accretion efficiency $\beta$ for various binary evolution sequences (symbols) and different physical assumptions, as function of the initial orbital period for $16 \mathrm{M}_{\odot}+15 \mathrm{M}_{\odot}$ systems. Triangles mark systems which have been calculated using rotational physics and the tidal model as proposed by Zahn (1977), which in systems marked by squares has been assumed to be one order of magnitude more efficient. Star symbols designate models by Wellstein et al. (2001) which have been computed without rotational physics and assumed to evolve conservatively unless a contact situation occurs. The dotted vertical line separates Case A systems (to the left) from Case B systems.

The problem of how wide the Case $\mathrm{C}$ channel is for such massive stars is difficult. Current single star models suggest no significant radius increase late during or after core helium burning at solar metallicity. On the other hand, Smartt's (2002) abundance analysis of Galactic supergiants suggests that they do not undergo "blue loop" evolution - which contradicts the current models. The problem is less severe at low metallicity, where many models predict a gradual radius increase during core helium burning.

It is even harder to produce Cyg X-1 type $\mathrm{BH}$ binaries, with black hole masses as high as $14 \mathrm{M}_{\odot}$. Brown et al. (2001) argue that this requires Case $\mathrm{C}$ evolution and an upturn of the initial-final mass relation for single stars above $\sim 60 \mathrm{M}_{\odot}$, as predicted by Langer (1987).

\section{Spins of compact objects and gamma-ray bursts}

The inclusion of rotationally enhanced magnetic fields in the evolution of massive single stars (Heger et al., this volume) has improved the agreement between observed $(15 \ldots 150 \mathrm{~ms})$ and predicted $(z 5 \mathrm{~ms})$ spin periods of young neutron stars - with the consequence that obtaining gamma-ray bursts from black hole for- 
mation in single stars (collapsars; Woosley \& Heger, this volume) seems difficult at present.

From Sections 3 and 4 above, we conclude that the initially more massive stars in massive close binaries are even less likely to produce a gamma-ray burst. First of all, they lose so much mass that even stars with a very large initial mass may not even form a black hole but rather a neutron star (see also Wellstein \& Langer 1999). And secondly, Figure 1 shows how drastic these stars are spun down as a consequence of their heavy mass loss. The $16 \mathrm{M}_{\odot}$ star in the computed binary system is expected to produce a neutron star with an initial spin period of more than one second!

The only way to avoid both drawbacks is to employ Case $\mathrm{C}$ evolution, which leads to a core evolution as in single stars. However, even then the CO-core of the star needs to be spun-up significantly to produce a collapsar and a gammaray burst - a possibility suggested by Brown et al. $(1999,2000)$ in the context of common envelope evolution and spiral-in. No detailed models for this scenario exist at present.

The initially less massive star in a massive binary, on the other hand, accretes large amounts of angular momentum and will thus acquire a larger core spin than a corresponding single star (see Fig. 1). It is thus conceivable that accretion stars are the progenitors of asymmetric supernova explosions and rapidly spinning compact objects. Those sufficiently massive to transform into a WolfRayet star during core helium burning, or those which lose their envelope in a reverse Case $\mathrm{C}$ mass transfer, may possess all required ingredients to produce a gamma-ray burst within the collapsar model.

\section{Progenitors of Type Ia supernovae}

Supernovae of Type Ia are supposed to occur in close binary systems where the accreting component is a $\mathrm{CO}$-white dwarf. The currently favoured scenario assumes that a white dwarf of initially less than $1 \mathrm{M}_{\odot}$ grows in mass due to quiescent nuclear shell burning of hydrogen and/or helium, which is accreted from a non-degenerate companion. In order to explode, it must reach the Chandrasekhar-mass, i.e. it needs to accrete at least $0.4 \mathrm{M}_{\odot}$. While single white dwarfs are slow rotators (Kawaler, this volume), the idea that Type Ia supernova progenitors are significantly spun-up due to accretion appears to be supported by recent measurements of the rotational velocity of the white dwarf in cataclysmic variables (Starrfield, this volume).

In order for the degenerate CO-core to grow in mass, accreting white dwarfs need to possess a helium burning shell source. However, like in AGB stars, which have a degenerate $\mathrm{CO}$-core in their center, the helium shell source in accreting white dwarfs tends to turn thermally unstable, causing large oscillations in nuclear energy production, as well as in the white dwarf radius (Fig. 4). It is unclear whether the binary system can survive these oscillations intact. However, Yoon et al. (2003) found that the consideration that angular momentum is accreted along with the matter by the white dwarf results in different shell source properties. The spin-up of the outer part of the white dwarf leads to a lower effective gravity and to a more relaxed helium shell source. Additionally, differential rotation leads to shear mixing at the location of the shell source, 


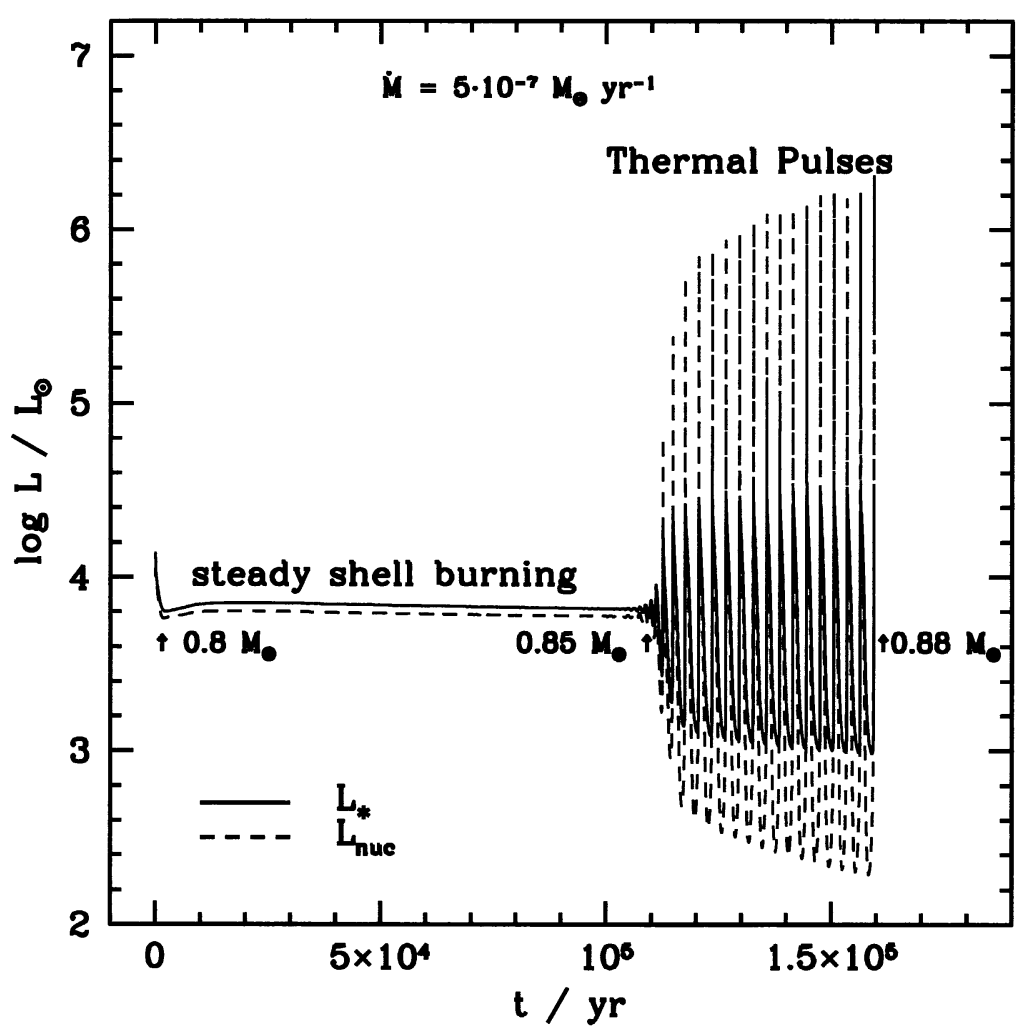

Figure 4. Stellar and nuclear luminosity as function of time for a $\mathrm{C} / \mathrm{O}$-white dwarf model starting at $0.8 \mathrm{M}_{\odot}$, which accretes helium at a constant rate of $5 \times 10^{-7} \mathrm{M}_{\odot} \mathrm{yr}^{-1}$ (Scheithauer 2000).

increasing its geometrical thickness and often turning it stable. This effect increases the likelihood of a given system to reach the Chandrasekhar-mass at all.

However, not only does the thickness of the helium shell source increase, but the chemical profiles through it become shallower (Fig. 5). As thus most of the helium burning occurs at a lower helium concentration, oxygen is produced at a much higher abundance than in corresponding non-rotating models, and carbon is produced correspondingly less. As the combustion of oxygen yields less energy than that of carbon, rotational effects influence the brightness of Type Ia supernovae. As the rotation rate depends on the amount of accreted material, and the average amount of accreted matter increases with the initial metallicity of the binary system (Langer et al. 2000), a drop of the brightness of Type Ia supernovae with higher metallicity — or an increase with redshift might occur. 


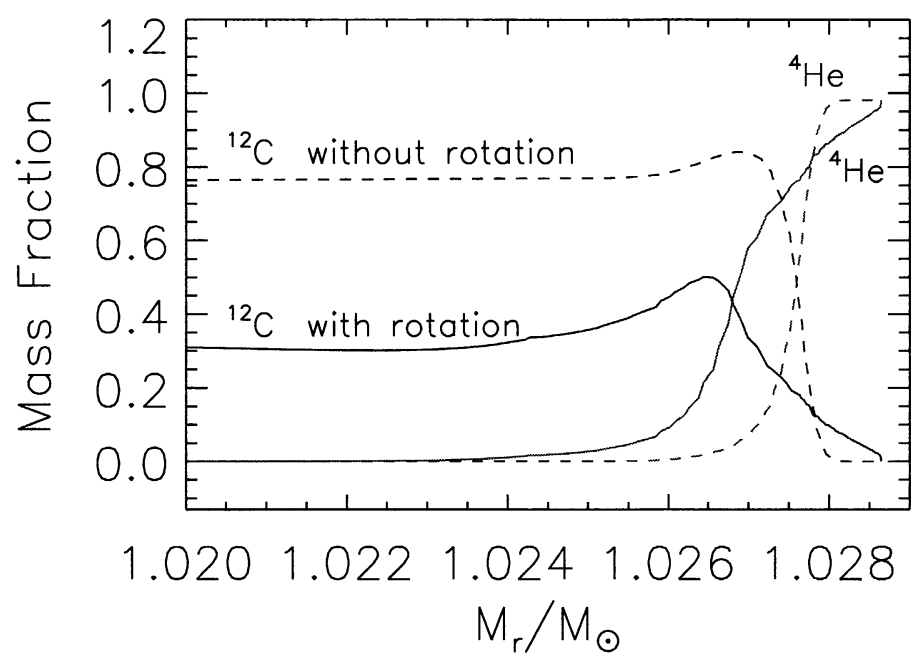

Figure 5. Helium and carbon mass fraction as function of the mass coordinate in a $\mathrm{CO}$ white dwarf which starts at $1 \mathrm{M}_{\odot}$ and accretes helium-rich matter at a rate of $10^{-6} \mathrm{M}_{\odot} \mathrm{yr}^{-1}$, at a white dwarf mass of $1.0286 \mathrm{M}_{\odot}$, computed with and without rotation (Yoon \& Langer 2002). Note that the carbon mass fraction $X_{\mathrm{C}}$ behind the shell source $\left(M_{\mathrm{r}}<1.025 \mathrm{M}_{\odot}\right)$ is lower in the rotating model $\left(X_{\mathrm{C}} \simeq 0.3\right)$ than in the non-rotating one $\left(X_{\mathrm{C}} \simeq 0.8\right)$, corresponding to an oxygen mass fraction of 0.7 rather than 0.2 .

Finally, rotation also affects the evolution of white dwarfs in the so called sub-Chandrasekhar mass scenario, as outlined by Yoon \& Langer (2003; also this volume).

\section{References}

Bohannan B., Conti P.S., 1976, ApJ 204, 797

Brown G.E., Lee C.-H., Bethe H.A., 1999, New Astron. 4, 313

Brown G.E., Lee C.-H., Wijers R.A.M.J., Lee H.K., Israelian G., Bethe H.A., 2000, New Astron. 5, 191

Brown G.E., Heger A., Langer N., Lee C.-H., Wellstein S., Bethe H.A., 2001, New Astron. 6, 457

Clark J.S., Goodwin S.P., Crowther P.A., Kaper L., Fairbairn M., Langer N., Brocksopp C., 2002, A\&A 392, 909

Fryer C.L., 2003, in A massive star odyssey: from main sequence to supernova, proc. IAU-Symp. 212, (San Francisco: ASP), p. 377 K.A. van der Hucht, A. Herrero \& C. Esteban, eds.

Heger A., Langer N., 2000, ApJ 544, 1016

Heger A., Langer N., Woosley S.E., 2000, ApJ 528, 368

Kaper L., Lamers H.J.G.L.M., Ruymaekers E., van den Heuvel E.P.J., Zuidervijk E.J., 1995, A\&A 300, 446

Langer N., 1987, A\&A 171, L1 
Langer N., 1997, in Luminous Blue Variables: Massive Stars in Transition, A. Nota, H.J.G.L.M. Lamers, eds, ASP Conf. Ser., Vol. 120, p. 332

Langer N., 1998, A\&A 329, 551

Langer N., Deutschmann A., Wellstein S., Höflich P., 2000, A\&A 362, 1046

Meynet G., Maeder A., 2000, A\&A 361, 101

Orosz J.A., 2003, in A massive star odyssey: from main sequence to supernova, proc. IAU-Symp. 212, (San Francisco: ASP), K.A. van der Hucht, A. Herrero \& C. Esteban, eds.

Packet W., 1981, A\&A 102, 17

Paczynski B., 1991, ApJ 370, 597

Petrovic J., Langer N., 2003, in A massive star odyssey: from main sequence to supernova, proc. IAU-Symp. 212, (San Francisco: ASP), K.A. van der Hucht, A. Herrero \& C. Esteban, eds.

Scheithauer S., 2000, Diploma thesis, Potsdam University

Smartt S.J., 2003, in A massive star odyssey: from main sequence to supernova, proc. IAU-Symp. 212, (San Francisco: ASP), K.A. van der Hucht, A. Herrero \& C. Esteban, eds.

Wellstein S., 2001, PhD thesis, Potsdam University

Wellstein S., Langer N., 1999, A\&A 350, 148

Wellstein S., Langer N., Braun H., 2001, A\&A 369, 939

Yoon S.-C., Langer N., 2003, A\&A 412, L53

Yoon S.-C., Langer N., 2002, in: The Physics of Cataclysmic Variables and Related Objects, ASP Conf. Ser. Vol 261, 2002, B.T. Gaensicke et al., eds., p 79

Yoon S.-C., Langer N., Scheithauer S., 2003, A\&A, in preparation

Zahn J.-P., 1977, A\&A 57, 383 